\title{
Synthetic strategies of multifunctional porphyrins as receptors
}

\author{
Hisanobu Ogoshi,* Yasuhisa Kuroda, Tadashi Mizutani, and Takashi Hayashi \\ Department of Synthetic Chemistry and Biological Chemistry, Faculty of Engineering, \\ Kyoto University, Sakyo-ku, Kyoto 606-01, Japan
}

\begin{abstract}
A series of multi-functional porphyrins 1-10 were prepared as a model receptor to clarify the mechanism of electron transfer reaction mediated by molecular recognition between reductase and mobile ubiquinone in membrane. Formation of complementary face-to-face complex is performed by multi-point hydrogen bonding between benzoquinones substituted with methoxy groups and particular porphyrins. Fluorescence from photo-chemically excited porphyrin is efficiently quenched by quinone derivative in the face-to-face adduct. Binding constants and thermodynamic parameters are discussed with structural changes in both quinones and host porphyrins. Newly prepared intrinsic chiral porphyrins with $C_{2}$ symmetry have been examined as a model receptor for amino acid esters. Thermodynamic investigation on the intermolecular interaction of chiral and reference porphyrins with amino acids enables us to estimate contribution of the respective interactions such as hydrogen bonding, coordination to the metal, and van der Waals contact. The host having the chiral cavity above and below the porphyrin plane shows high diastereomeric selectivity towards amino acids with bulky residue.
\end{abstract}

\section{INTRODUCTION}

A variety of porphyrins and metalloporphyrins have been synthesized for model compounds to understand the functions of heme proteins, heme enzymes, chlorophyll ensemble and vitamin $B_{12}$. In addition to those models of biologically important elements, synthetic strategies of multifunctional porphyrins as functional dyes have been extended to clarify the mechanism of molecular recognition of naturally occurring small organic substrates such as amino acids, saccharides, and nucleotides. Most prominent advantages of porphyrin framework are summarized as follow: i) Facile introduction of a variety of functional groups at the peripheral positions. ii) Chemical changes in the macro cyclic ring is easily monitored by molecular spectroscopic methods. iii) Complex formation with many metal ions.

We have synthesized the first strapped porphyrins and cyclophane porphyrins by utilizing the porphyrins having divergent functional groups at earlier time $(1,2)$. If convergent functional groups are required for interaction sites in the porphyrin framework, synthesis of the meso aryl substituted porphyrin like precursor of the picket-fence porphyrin is one of promising synthetic approach (3). The convergent functional groups could be fixed by two synthetic methods; The first one is an introduction of the alkyl groups at the $\beta$-position of pyrrole rings and the second one is condensation of pyrrole with the substituted naphthaldehyde. Atropisomers obtained from two syntheses are not interconvertible at the ambient temperature due to steric constraint at the meso positions.

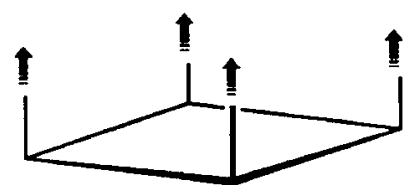

convergent form

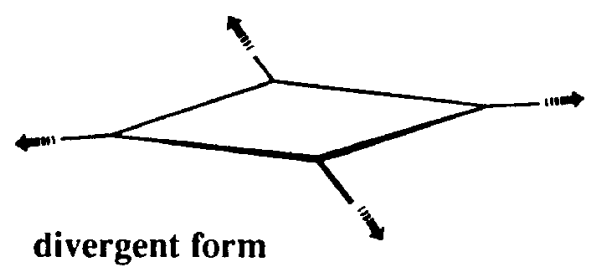

divergent form 
The axial chirality with $C_{2}$ symmetry in the terphenyl derivatives has prompted us to prepare new chiral porphyrins. Terminal ortho or meta-substituted phenyl groups are in the same plane and substituents have a trans conformation. Substituents of the middle phenyl ring are asymmetric relative to the bisected plane involving the two terminal phenyl groups as shown below.

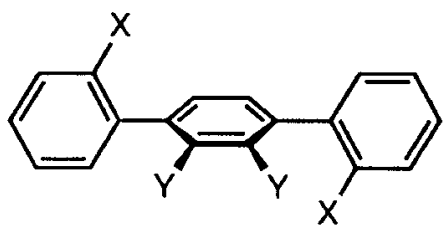

\section{MODEL RECEPTORS FOR QUINONES}

Cis-bifunctional receptors 2 and 3 bind to quinones and nucleobase through two points hydrogen bonding $(4,5)$. The porphyrin 3 acts as preferable receptor to plastoquinone type substrates. Cofacial molecular recognition operating in these complexes are due to electronic effect of substituents of quinones, charge transfer between host and guest, and steric repulsion. Complementarity of molecular recognition has been examined for the pair of ubiquinone analogues and multifunctional porphyrins atropisomers 1 having four convergent hydroxyl groups $(6,7)$. Hydrogen bonds between the $\mathrm{OH}$ groups of host and carbonyls and

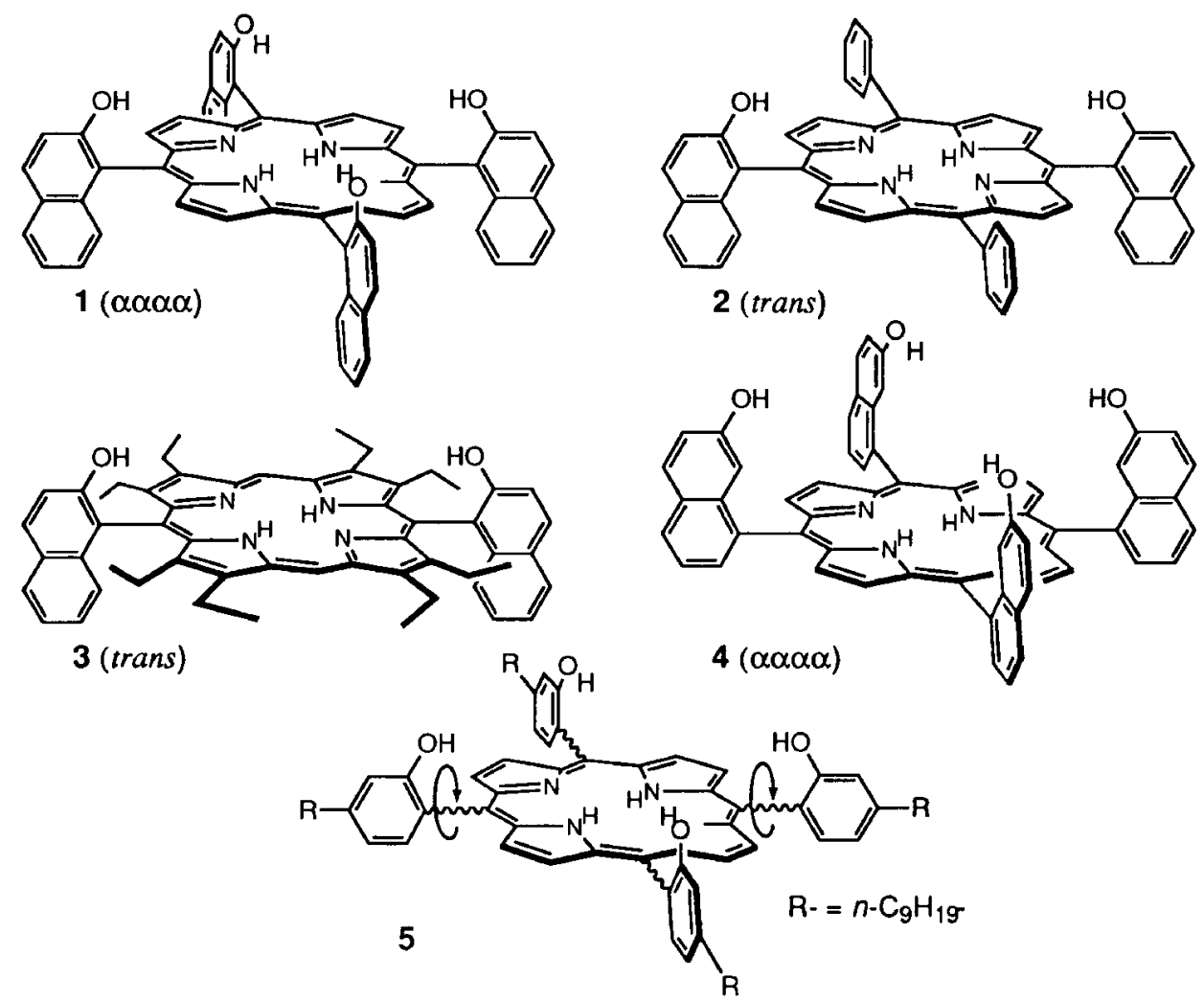

methoxy groups constitute complementary recognition sites. Table 1 shows comparison of the binding constants of multi-functional porphyrins with quinone derivatives and the associated thermodynamic parameters. Binding of quinone derivatives is clearly dependent on numbers and positions of the methoxy groups. The crystal structure of $\alpha \alpha \alpha \alpha$-isomer 1 and tetramethoxy-p-benzoquinone (TMBQ) shows formation of the face-to-face complex. Convergent but more mobile interaction sites in host 4 dramatically decreases binding constant of TMBQ (8). Fluorescence quenching measurements evidently indicate electron transfer from photochemically excited porphyrin to the quinone involving cofacial complex as major process. 
TABLE 1. Binding affinities $\left(K_{\mathrm{a}}, \mathrm{M}^{-1}\right)$ for ubiquinone analogues in toluene at $25^{\circ} \mathrm{C}^{a}$

\begin{tabular}{llllll}
\hline & 860 & 13,000 & 35,000 & 610,000 \\
\hline $1(\alpha \alpha \alpha \alpha)$ & 220 & 320 & 220 & 410 & 210 \\
$2(c i s)$ & 98 & 390 & 250 & 810 & 570 \\
\hline$(\alpha \alpha \alpha \alpha)$ & 150 & 20 &
\end{tabular}

"Binding alfinities were determined from electronic absorption studies at $584 \mathrm{~nm}$.

Introduction of long alkyl chains at the para position of the meso-2-hydroxyphenyl groups has improved solubility dramatically in organic solvents comparing with that of the parent porphyrin. Time course of atropisomerization indicates that dynamic induced fit-type of molecular recognition takes place to lead preferable complex formation with quinone as are seen in Figure 1. Marked change of statistical ratio of interconvertible atropisomers 5 was induced by addition of ubiquinone analogues (9).

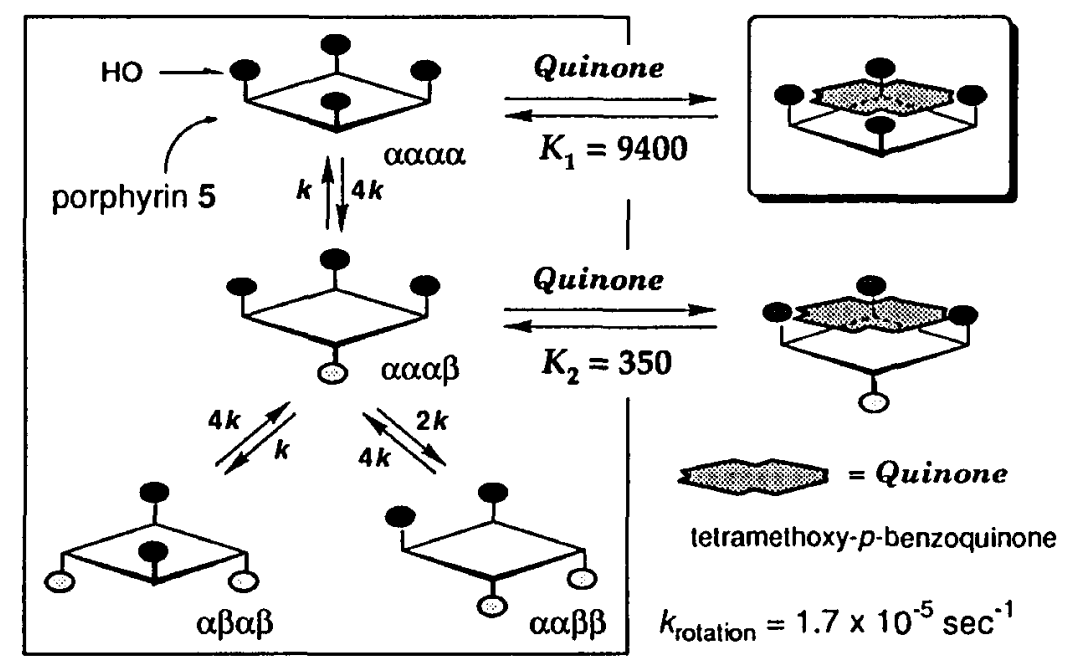

Figure 1. Schematic representation of dynamic process in 5-quinone adduct

\section{MODEL RECEPTOR FOR AMINO ACID ESTERS}

Trans-bifunctional metalloporphyrin 6 interacts reversibly with amino acid esters through metal coordination of $-\mathrm{NH}_{2} \bullet \mathrm{Rh}$ (III) or $\mathrm{Zn}$ (II) and $-\mathrm{OH} \cdots \cdot \mathrm{RO}_{2} \mathrm{C}$ - hydrogen bonding. The host-guest chemistry of particular pair has been clarified by thermodynamic characterization and induced circular dichroism due to the fixation of chiral amino acid at near proximity of chromophores (10-14).

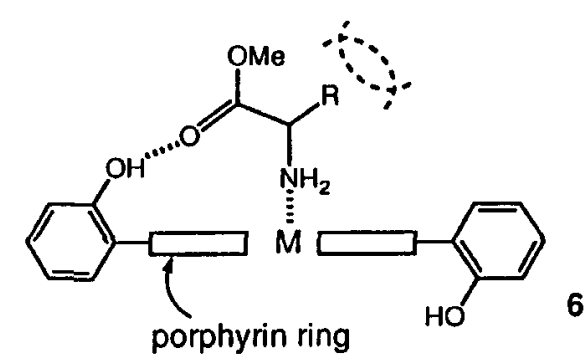


Intrinsic chiral porphyrins 7 with $\mathrm{C}_{2}$ symmetry are constructed with the trans-bifunctional aromatic fragments bisected by an asymmetric porphyrin (15). Contribution of thermodynamic parameters, free energy change, enthalpy change and entropy change for complex formation were approximately separated into metal coordination, hydrogen bonding and Van der Waals interaction by comparison with those of the reference porphyrin hosts as shown in Table 2.

TABLE 2. Binding Constants $(K)$ of Amino Acid Esters to Zinc Porphyrins ${ }^{a}$

\begin{tabular}{lcccc}
\hline & \multicolumn{4}{c}{$K\left(\mathrm{M}^{-1}\right)$} \\
$\mathbf{7 a}$ & $\mathbf{7 b}$ & $\mathbf{7 c}$ & $\mathbf{7 d}$ \\
\hline L-Ala-OMe & 1590 & 3460 & 720 & 740 \\
D-Ala-OMe & 1420 & & & \\
L-Leu-OBzl & 3450 & 10100 & 560 & 1060 \\
D-Leu-OBzl & 1540 & & & \\
L-Ser-OBzl & 1340 & 2400 & 920 & 480 \\
D-Ser-OBzl & 2840 & & &
\end{tabular}

${ }^{a}$ At $15^{\circ} \mathrm{C}$ in chloroform. Standard deviations were within $4 \%$.

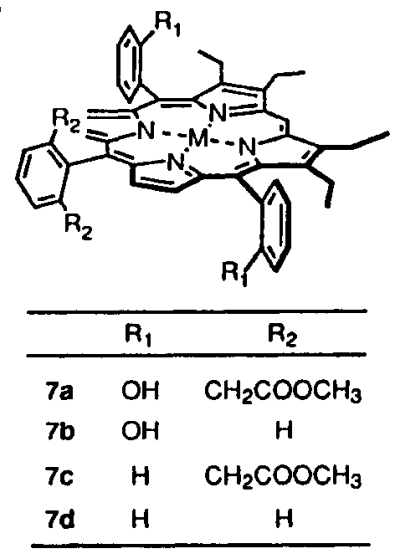

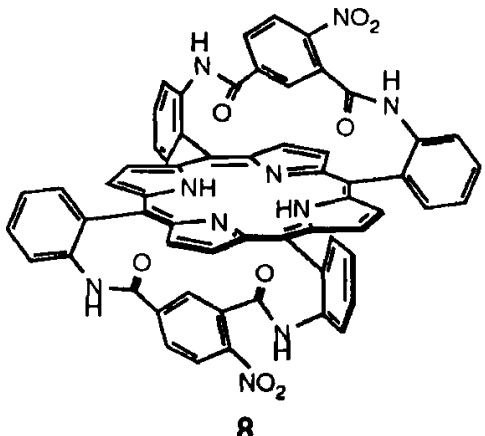

8

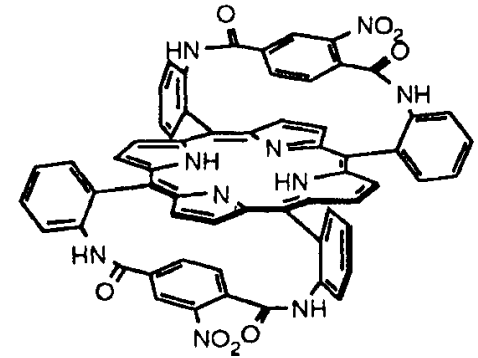

9

Novel intrinsic chiral porphyrins, 8 and 9 , were prepared from condensation of $\alpha, \alpha, \beta, \beta$-tetrakis(2aminophenyl)porphyrin and asymmetric phthaloyl dichloride $(16,17)$. Chiral recognition operating in the space of doubly bridged 8 are due to the coordination of $\mathrm{NH}_{2}$ to $\mathrm{Zn}$, hydrogen bonding between ester carbonyl of amino acid and bridge amide proton, and van der Waals contact of amino acid residues. Zinc complex of para bridged porphyrin 9 constitutes smaller space for axial ligation. Interaction of bulkier amino acid residue like phenylalanine ester with zinc porphyrin 8 shows higher diastereomeric excess as seen in Table 3. Electronic effect of the nitro group in the bridged phenyl ring affects the acidity of the amide proton, and the more acidic amide proton in turn increases the binding of substrates toward the host 8 as shown in Table 3.

TABLE 3. Binding Constants $\left(K, M^{-1}\right)$ for the Host-Guest Complex in $\mathrm{CH}_{2} \mathrm{Cl}_{2}$ at $278 \mathrm{~K}$

\begin{tabular}{|c|c|c|c|}
\hline $\begin{array}{c}\text { porphyrin } 8 \\
(+) \cdot Z n\end{array}$ & $2.3 \times 10^{5}$ & $6.1 \times 10^{5}$ & $1.7 \times 10^{5}$ \\
\hline$(-) \cdot \mathrm{Zn}$ & $3.9 \times 10^{6}$ & $7.4 \times 10^{6}$ & $8.7 \times 10^{5}$ \\
\hline$K(-) / K(+)$ & 17.0 & 12.1 & 5.1 \\
\hline $\begin{array}{l}\Delta \Delta G^{\circ} \\
(\mathrm{kcal} / \mathrm{mol})\end{array}$ & 1.57 & 1.37 & 0.90 \\
\hline
\end{tabular}




\section{PORPHYRINS FOR THERAPEUTIC CHEMISTRY}

Porphyrins and their metal complexes substituted with carboranyl groups have been prepared for BNCT (Boron neutron capture therapy) $(18,19)$. Introduction of large carboranyl groups has a high possibility of neutron capture, but it turns out to decrease solubility in water. Functional dyes were soluble in water with polar organic solvent like THF or DMSO. Simple condensation reaction of pyrrole and pdihydroxyborylbenzaldehyde protected with 2,2-dimethylpropane-1,3-diol afforded porphyrin having four phenylboronic acids in moderate yield. However, the obtained acids are slightly soluble in water. Addition of maltitol enhances its solubility in water due to specific interaction of the sorbitol moiety with the boronic acid and increase of water affinity with aid of the glucose moiety. Porphyrin derivatives have been utilized as chemical probe for diagnosis of earlier lung cancer and photo sensitizer. The present new derivative 10 having boronic acid may provide us a new chemical probe for diagnosis and BNCT (20).

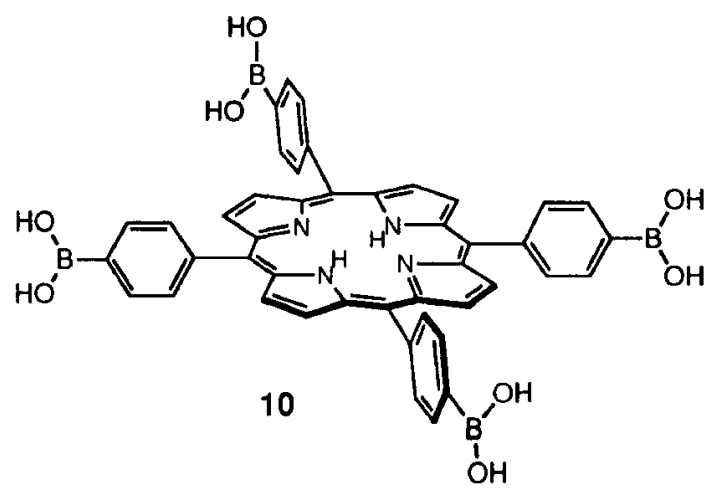

\section{Acknowledgment}

This work was supported by a Grant-in-Aid for Specially Promoted Research (No. 04101003) from the Ministry of Education, Science, and Culture, Japan.

\section{REFERENCES}

1. H. Ogoshi, H. Sugimoto and Z.-i. Yoshida. Tetrahedron Lett.. 4477 (1976).

2. H. Ogoshi, H. Sugimoto and Z.-i. Yoshida. Tetrahedron Lett. 169 (1977).

3. J. P. Collman, R. R. Gagne, C. A. Reed, T. R. Halbert, G. Lang and W. T. Robinson. J. Am. Chem. Soc. 97, 1427 (1975).

4. Y. Aoyama, M. Asakawa, Y. Matsui and H. Ogoshi. J. Am. Chem. Soc. 113, 6233 (1991).

5. T. Hayashi, T. Miyahara, Y. Kato and H. Ogoshi. unpublished results

6. T. Hayashi, T. Miyahara, N. Hashizume and H. Ogoshi J. Am. Chem. Soc. 115, 2049 (1993).

7. T. Hayashi, T. Miyahara, Y. Aoyama, M. Kobayashi and H. Ogoshi. Pure Appl. Chem. 66, 797 (1994).

8. T. Hayashi, T. Miyahara, Y. Aoyama, M. Nonoguchi and H. Ogoshi. Chem. Lett. 1749 (1994).

9. T. Hayashi, T. Asai, H. Hokazono and H. Ogoshi. J. Am. Chem. Soc. 115, 12210 (1993).

10. Y. Aoyama, A. Yamagishi, M. Asakawa, H. Toi and H. Ogoshi. J. Am. Chem. Soc. 110, 4076 (1988).

11. Y. Aoyama, M. Asakawa, A. Yamagishi, H. Toi and H. Ogoshi. J. Am. Chem. Soc. 112, 3145 (1990),

12. H. Ogoshi, H. Hatakeyama, K. Yamamura and Y. Kuroda. Chem. Lett. 51 (1990).

13. H. Ogoshi, H. Hatakeyama, J. Kotani, A. Kawashima and Y. Kuroda. J. Am. Chem. Soc. 113, 8181 (1991).

14. T. Mizutani, T. Ema, T. Yoshida, T. Renne and H. Ogoshi. Inorg. Chem. 33, 3558 (1994).

15. T. Mizutani, T. Ema, T. Tomita, Y. Kuroda and H. Ogoshi. J. Am. Chem. Soc. 116, 4240 (1994).

16. Y. Kuroda, Y. Kato, T. Higashioji and H. Ogoshi. Angew. Chem., Int. Ed., Engl. 32, 723 (1993).

17. Y. Kuroda, Y. Kato, M. Ito, J. Hasegawa and H. Ogoshi. J. Am. Chem. Soc. in press.

18. M. Miura, D. Gabel, G. Oenbrink and R. G. Fairchild. Tetrahedron Lett. 31, 2247 (1990).

19. S. B. Kahl and M.-S. Koo. J. Chem. Soc., Chem. Commun. 1769 (1990).

20. H. Toi, Y. Nagai, Y. Aoyama, H. Kawabe, K. Aizawa and H. Ogoshi. Chem. Lett. 1043 (1993). 